\title{
Welcome to the 11th volume of Epigenomics
}

\author{
Lucy Chard*,1 \\ ${ }^{1}$ Future Medicine, Future Science Group Ltd, London, UK \\ *Author for correspondence: I.chard@futuremedicine.com
}

Foreword by Lucy Chard: Welcome to the 11th volume of Epigenomics. I would like to take this opportunity to wish all of our readers a Happy New Year. In this Foreword, I shall be taking a look back at some of the journal highlights of 2018.

Over the past year, we have seen some great strides in the development of epigenetics for medical applications. However, this advancing field, while niche, still needs to overcome a number of obstacles before we see a more widespread application of this technology. It has been a privilege for Epigenomics to publish work in this fascinating field, which continues to be of the highest quality.

\section{Article highlights}

We have published a number of outstanding Research Articles in the journal this year. Therefore, I would like to draw your attention to some of the particular highlights of 2018.

At the time of writing our most read article from 2018 was a Research Article entitled 'A novel cell-type deconvolution algorithm reveals substantial contamination by immune cells in saliva, buccal and cervix' by Andrew E Teschendorff and Shijie C Zheng from the Department of Women's Cancer, University College London and the University of Chinese Academy of Sciences, respectively [1]. The research paper was dedicated to developing and validating a new DNA methylation reference and algorithm to be used in complex tissues containing different types of cells: epithelial cells as well as immune and nonimmune stromal cells. Using the reference, Zheng et al. were able to confirm that each of the cervical, salvia and buccal tissues exhibit substantial variation in the total immune cell (IC) fraction, with a large proportion of the samples containing more than 70\% ICs [1].

This article is closely followed by another research paper: 'Exercise training alters the genomic response to acute exercise in human adipose tissue' by Fabre et al. [2], from the University of Copenhagen, published online in April 2018. This study showed that significant epigenomic changes occur in adipose tissue after exercise, tested using nucleases and DNA-modifying enzymes to target exercise responsive genes. This has laid the groundwork for further study in this area to establish entire causality and physiological relevance [2].

Researchers from Chulalongkorn University published the paper: 'Alu siRNA to increase Alu element methylation and prevent DNA damage' [3]. This research aimed to develop a technology to allow DNA methylation at Arthrobacter luteus (Alu) restriction endonuclease elements to increase genomic stability by reducing endogenous DNA damage. This research could have implications in the prevention of cancer and aging.

Another of the most highly read articles of 2018 entitled 'Stability of global methylation profiles of whole blood and extracted DNA under different storage durations and conditions' was authored by Li $e$ t al. The researchers from the Medical College of Wisconsin, USA used blood and DNA samples stored from 1996 at $4^{\circ} \mathrm{C}$ to produce libraries in 2016. The results from this Research Article support the idea that DNA samples can still be viable after many years of storage under the right conditions [4].

Epigenomics publishes a range of article types meaning that we are able to highlight some particularly interesting and novel topics, such as in this Editorial: 'Folate and epigenetics: why we should not forget bacterial biosynthesis' by Dieuwertje E Kok, Wilma T Steegenga and Jill A McKay from Wageningen University and Research in the Netherlands [5]. This editorial article was published in the September issue and has already received a high number of downloads. They propose that bacterial folate synthesis provides an additional source of folate, this brings important impacts for the field [5]. This piece explores the role of folate in epigenetic regulation and possible disease outcomes, posing many important questions for future research. 

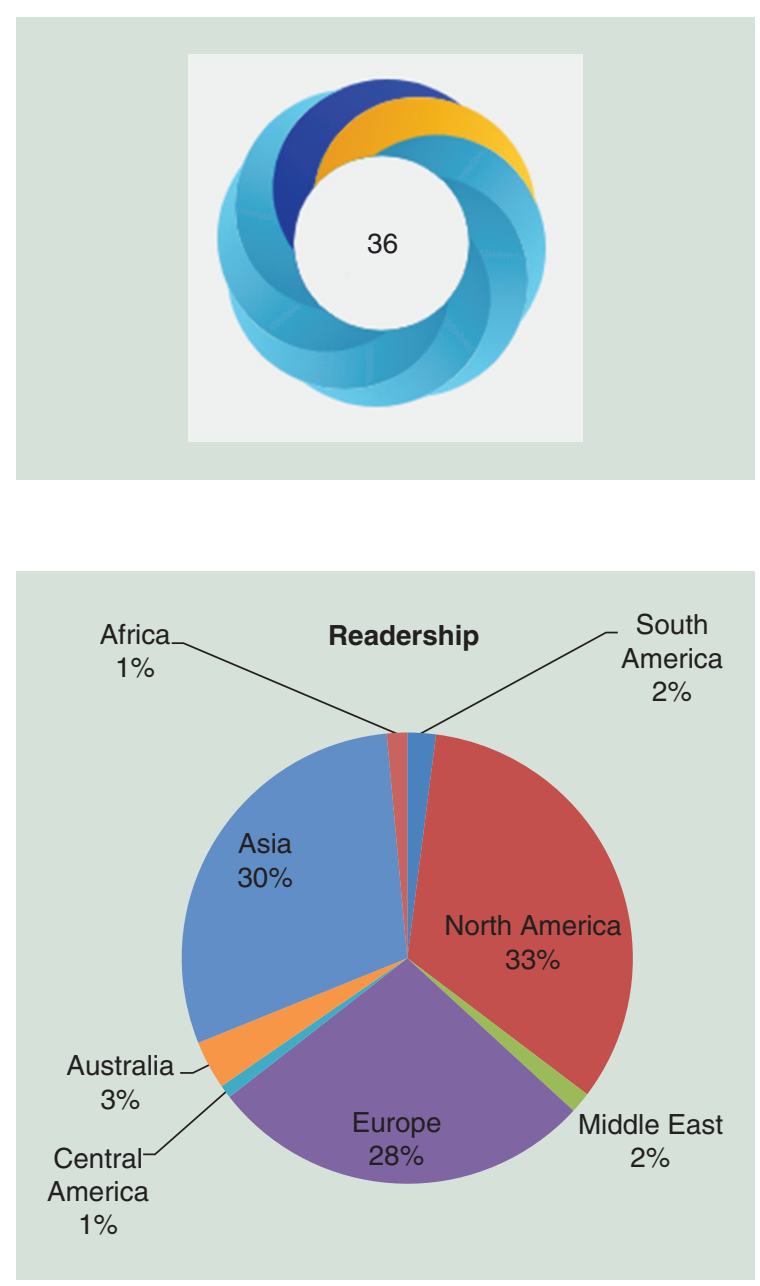

Figure 1. The colors of the wheel represent kinds of sources. Here, the yellow represents a blog, the dark blue represents Facebook and the light blue represents Twitter users.
Figure 2. Proportion of readership demographics for Epigenomics in 2018.

\section{Altmetrics}

Altmetrics is a powerful way to measure the impact of our publications. It collates information from multiple different sources on different platforms to show the way in which the research is being broadcast across the world.

The most prominent example of this from 2018 has been the Research Article by de Vega et al. entitled: 'Integration of DNA methylation \& health scores identifies subtypes in myalgic encephalomyelitis/chronic fatigue syndrome' [6]. This has received an Altmetrics score of 36 since its publication in April. A combination of tweets, blogs and other social media influences has engaged readers from throughout Europe, America and Australia. In this study, the team from University of Toronto, Ontario, Canada, used DNA methylation profiles to characterize myalgic encephalomyelitis/chronic fatigue syndrome. This condition is notoriously difficult to diagnose due to the high variability in symptoms and similarities to other diseases such as thyroid problems [6].

The Altmetrics features a useful graphic to visualize the 'Altmetric Attention Score' for a research output (Figure 1). The score is derived from an automated algorithm, and represents a weighted count of the amount of attention received for a research output [7].

\section{Geographical spread of our contributors}

As the diversity of the research community continues to grow globally, we have also seen changes in the readership of Epigenomics from 1 year to the next. This past year, our content was most read by experts in North America $(33 \%)$. Asia also makes up a large proportion of our readership with $30 \%$. A high proportion of our readers come from Europe with 28\% (Figure 2). I am also pleased to see an increase in the numbers of readers from Africa and Australia. The increase in readership across Asia from last year is exciting in showing the changing research climate. Epigenomics continues to welcome both readers and content from across the world. 
Figure 3. Proportion of authorship demographics for Epigenomics in 2018.

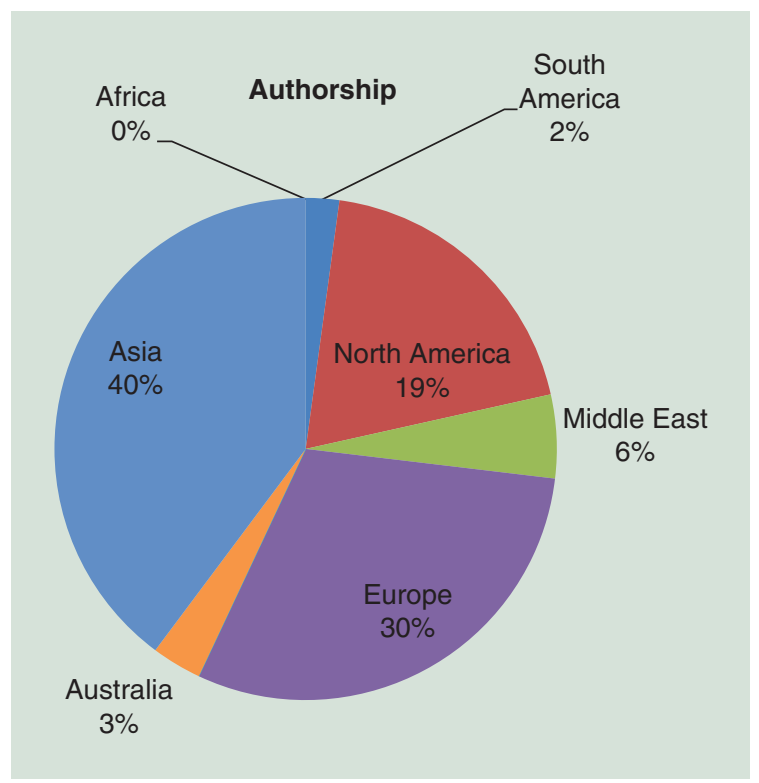

It is also interesting to see the proportion of authors from different countries and how this is comparable to the readership. With a higher number of authors from Asia and the Middle East being published (Figure 3).

\section{Twitter}

As many of our readers will be aware, Epigenomics has a Twitter account. We use Twitter to showcase content highlights, journal updates and key news articles from the field. With nearly 2000 followers, this is an excellent way to feature the journal and its contents on a global platform, as well as sharing topical news as it happens. It has been a pleasure to engage with our authors and readers in a more informal setting by using twitter and I encourage anyone on Twitter to follow us (@fsgepi) [8].

\section{Conclusion}

I would like to take this opportunity to thank everyone who has helped with the success of Epigenomics in 2018. Special thanks go to the Editorial Board members who are always available for support and advice. We welcome unsolicited Research, Review and Editorial article proposals, and would be delighted to hear from you if you are interested in submitting to the journal to continue on this success into 2019.

Many thanks for your continuing interest and support for Epigenomics, I look forward to collaborating with many of you over the coming year.

\section{Financial \& competing interests disclosure}

$\mathrm{L}$ Chard is an employee of Future Medicine Ltd. The author has no other relevant affiliations or financial involvement with any organization or entity with a financial interest in or financial conflict with the subject matter or materials discussed in the manuscript apart from those disclosed.

No writing assistance was utilized in the production of this manuscript.

\section{References}

1. Zheng S, Webster A, Dong D et al. A novel cell-type deconvolution algorithm reveals substantial contamination by immune cells in saliva, buccal and cervix. Epigenomics 10(7), 925-940 (2018).

2. Fabre O, Ingerslev LR, Garde C, Donkin I, Simar D, Barrès R. Exercise training alters the genomic response to acute exercise in human adipose tissue. Epigenomics 10(8), 1033-1050 (2018).

3. Patchsung M, Settayanon S, Pongpanich M, Mutirangura D, Jintarith P, Mutirangura A. Alu siRNA to increase Alu element methylation and prevent DNA damage. Epigenomics 10(2), 175-185 (2018).

4. Li Y, Pan X, Roberts M et al. Stability of global methylation profiles of whole blood and extracted DNA under different storage durations and conditions. Epigenomics 10(6), 797-811 (2018). 
5. Kok D, Steegenga W, McKay J. Folate and epigenetics: why we should not forget bacterial biosynthesis. Epigenomics 10(9), 1147-1150 (2018).

6. de Vega W, Erdman L, Vernon S, Goldenberg A, McGowan P. Integration of DNA methylation \& health scores identifies subtypes in myalgic encephalomyelitis/chronic fatigue syndrome. Epigenomics 10(5), 539-557 (2018).

7. Altmetric.com. (2018). Altmetric Explorer. www.altmetric.com/explorer/outputs?journal_id\%5B\%5D=4f6fa6163cf058f6100072c9\&p ublished_after=2018-01-01\&published_before=2018-10-22\&show_details=39328079

8. Twitter.com. (2018). Epigenomics Journal (@fsgepi) on Twitter. https://twitter.com/fsgepi?lang=en-gb 